\title{
Successful Ablation of Ventricular Arrhythmia Without Fluoroscopy Guided by Carto 3 System in Pregnant Woman Without Structural Heart Disease
}

\author{
Ayan Abdrakhmanov $^{1,2}{ }^{\circledR}$, Aliya Smagulova ${ }^{2 *}{ }^{\circledR}$ Bayan Ainabekova $^{2}$
}

\begin{abstract}
Introduction: Arrhythmias can take place in any period of pregnancy. In addition, the incidence of life-threatening ventricular arrhythmias in a pregnant woman without the organic pathology of the heart is rare. Interventional treatment should be carried out in cases of severe arrhythmias and drug resistance. The radiation exposure during ablation carries a potentially harmful effect on the mother and fetus although data on the zero-fluoroscopy ablation of arrhythmias in pregnant women is limited.

Case Presentation: A 26-year-old female without structural heart disease at the gestation period of 26-28 weeks was admitted to a hospital due to severe symptoms of ventricular tachycardia (VT) and premature ventricular contractions. In this regard, the conservative therapy of $\beta$-blockers was ineffective and accompanied by a decrease in blood pressure to $60 / 40 \mathrm{~mm} \mathrm{Hg}$.

Results: An intracardiac electrophysiological study and non-fluoroscopic catheter ablation were carried out considering the drug refractory and severe symptoms of VT. The ablation of the arrhythmia substrate was successfully performed using the Carto 3 System without fluoroscopy. Based on the results, the procedure was not associated with any maternal or fetal complications.

Conclusions: In general, the non-fluoroscopic catheter ablation guided by electro-anatomical mapping and navigation systems is safe and applicable in the treatment of pregnant women with severe types of arrhythmias.

Keywords: Pregnancy, Ventricular tachycardia, Catheter ablation
\end{abstract}

\section{Introduction}

Arrhythmias may occur during the gestation period. In their study on 136422 pregnancy-related admissions, Li et al (1) showed a prevalence of 166 in 100000 admissions due to arrhythmia. The most common types of arrhythmias were associated with the changes in sinus rhythm, namely, $60 \%$ of the total arrhythmia occurrence. In addition, premature contractions and supraventricular tachycardia (VT) were reported in 19\% and $14 \%$ of arrhythmia admissions. However, atrial fibrillation, VT, and atrial-ventricular block were rare accounting for only $1 \%$ of all admissions. Normal physiologic changes occurring during pregnancy may trigger arrhythmias due to increases in plasma volume (40\%-50\%), cardiac output (30\%-50\%), and the heart rate (30\%-50\%) during the gestation period. These factors increase the stretching of cardiac chambers, leading to the mismatch of membrane depolarization/refractory period and being the proarrhythmic factors in gestation $(2,3)$. The congenital and structural heart diseases of women and the history of arrhythmias increase the risk of new-onset and recurring arrhythmia episodes during pregnancy $(4,5)$. Nonetheless, the management of arrhythmias in pregnant women has some difficulties and the data of efficiency and safety of the pharmacological therapy of pregnant women is limited. Further, the majority of anti-arrhythmic drugs can cause risks for maternal and fetal side effects $(6,7)$. The catheter ablation of arrhythmias guided by electroanatomic 3D mapping systems has demonstrated similar effects such as conventional fluoroscopy-based ablation methods $(8,9)$. There are some small series and isolated case reports of the successful zero-fluoroscopy catheter ablation of different types of arrhythmias in pregnant women (10-12). The current study aimed to present a case of VT during pregnancy and its treatment with the electroanatomic 3D mapping system.

\section{Case Presentation}

A 26-year-old female at the gestation period of 26-28 weeks complained of heart palpitations, fainting during the palpitation, and dizziness referring to the hospital. In the disease history, arrhythmia took place within 3 months. Conservative therapy by $\beta$-blockers turned out to be ineffective and accompanied by a decrease in the blood pressure to $60 / 40 \mathrm{~mm} \mathrm{Hg}$. Therefore, the patient was examined and the results of general clinical tests and analysis of thyroid function were within normal limits. The 12-lead electrocardiogram recorded frequent 
premature ventricular contraction, along with short and unstable runs of VT (Figure 1). Moreover, 14368 monofocus premature ventricular contractions, 1610 single extrasystoles, 355 paired extrasystoles, and 11975 episodes of bigeminy were registered at 24-hour Holter monitoring, followed by recording 24 runs of VT with a maximum heart rate of $254 \mathrm{bpm}$. The largest length of VT consisted of 6 complexes. The organic pathology of the heart was not detected at echocardiography.

An intracardiac electrophysiological study and a nonfluoroscopic radiofrequency catheter ablation were conducted by taking into consideration the drug refractory and severe symptoms of VT. A reference electrode (BW Ref Star QwikPatch) was placed under the patient. The right femoral vein was punctured according to the Seldinger's method using the local anesthesia with Sol. Lidocaini 1,0\%-30,0 mL. In addition, the irrigated/mapping Smart Touch Navigation catheter was injected via the introducer $8 \mathrm{Fr}$. Then, the 3D map of the right heart was constructed by means of fast anatomical mapping (FAM) (Figure 2).

Further, an activation map was created in the right ventricular output section. Furthermore, the zone of the earliest activation was found: the right ventricular outflow tract (absolute pre-emphasis - $30 \mathrm{~ms}$ ) on the posterolateral area. Three radio-frequency influences were implemented (Power $=30 \mathrm{~W}, \mathrm{t}=40-45^{\circ} \mathrm{C}, \mathrm{I}=115$ $125 \Omega$, and the duration of 1 minute) with the criterion of "warming up" and cupping VT. VT did not recur after the catheter ablation.

The further course of pregnancy was normal and the 40-week pregnancy ended in spontaneous childbirth, a healthy child was born weighing $3250 \mathrm{~g}$. The recurrence of arrhythmias was not observed for 1 year after the operation.

\section{Discussion}

The incidence of VT during pregnancy is rare. However, despite its rarity, ventricular arrhythmia often leads to the death of the mother and fetus in patients with organic pathology of the heart $(1,7)$. Moreover, the mechanism of life-threatening ventricular arrhythmias in a woman without structural heart disease is unknown. Antiarrhythmic drug therapy is required in cases of severe symptoms or haemodynamically unstable arrhythmia $(6,7)$. The right ventricular outflow tract VT during the gestation period may be a severe condition with unstable blood pressure and fetal distress, which requires emergency treatment (13).

Catheter ablation is suggested in drug-refractory and poorly tolerated arrhythmias (10-12). To overcome the adverse effect of radiation, catheter ablation was performed using navigation systems that allow observing the anatomy of the heart and the location of the catheters inside the heart without the use of fluoroscopy. The effectiveness and safety of the zero-fluoroscopy ablation of the idiopathic VT were shown in a multicenter

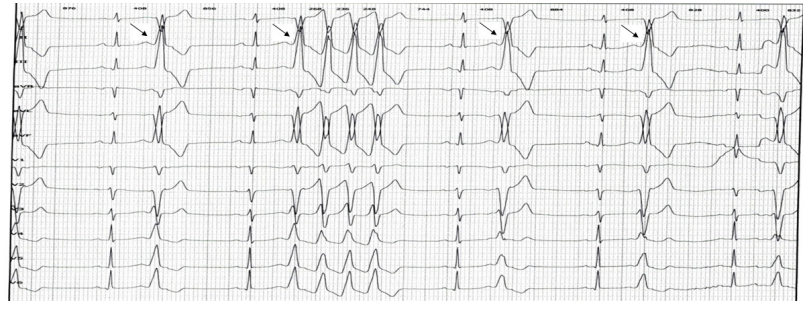

Figure 1. The 12-Lead Electrocardiogram Showing Frequent Premature Ventricular Contraction, Along With Short and Unstable Runs of Ventricular Tachycardia With the Morphology of the Left Bundle Branch Block.

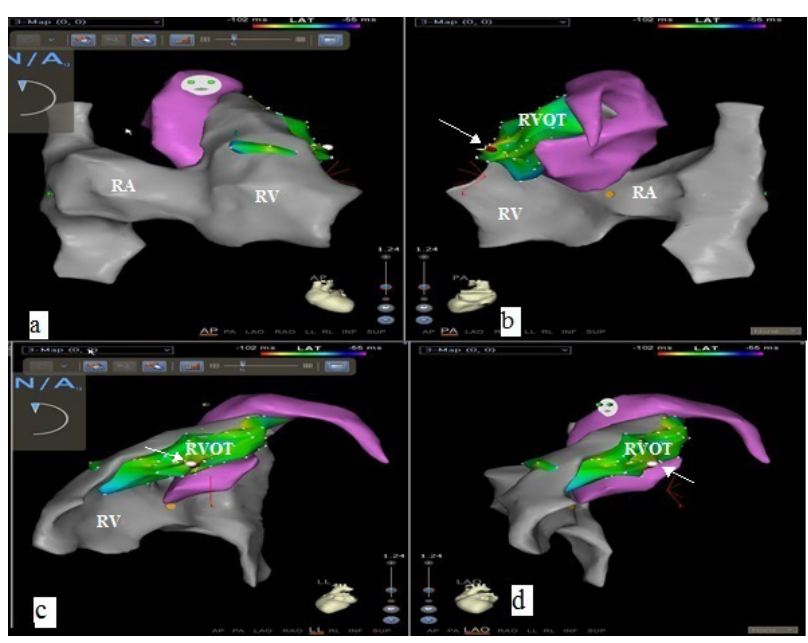

Figure 2. The Constructed 3D Map of the Right Atrium, Right Ventricular and Aortic Root in Anteroposterior (a), Posteroanterior (b), and Left Lateral (c) and Left Anterior Oblique (d) Views

Note. Activation map is showing the earliest activation site in the right ventricular outflow tract. The yellow dot indicates the His bundle. The red dot indicates the place of radiofrequency application. Pink color denotes aortic root, grey color defines the right atrium and right ventricular.

comparative study $(\mathrm{n}=489)$ in China (14). Additionally, the EnSite NavX 3D mapping system was used in all cases of zero-fluoroscopy ablation $(n=163)$. The data of VT ablation in the gestation period was limited since the pregnant women were not enrolled in this study. Prior reports on pregnant women have described successful non-fluoroscopic ablation procedures of supraventricular tachycardia (10-12). In one case, pregnant women with drug-refractory VT required cardioversion and emergency cesarean section (13). To the best of our knowledge, there was only one case in the literature regarding the successful use of the interventional treatment of life-threatening ventricular arrhythmias during the gestation period (15). This case described the ablation of ventricular arrhythmias with the pathological source of excitation localized in the outflow tract of the right ventricular guided by the EnSite NavX system without any use of fluoroscopy. The further gestation period was without the recurrence of arrhythmia and the woman was delivered by the elective cesarean section.

The current study presented the rare case of non- 
fluoroscopic catheter ablation usage in the new-onset of ventricular arrhythmias with a pathological source of excitation localized in the outflow tract of the right ventricular in pregnant women without the organic pathology of the heart. Anti-arrhythmic therapy was inefficient and associated with hypotension in our patient. On the other hand, the catheter ablation of the arrhythmia substrate was successfully performed using Carto 3 System without fluoroscopy. The procedure was not associated with any maternal or fetal complications. In our case, the woman delivered on term by spontaneous delivery. The operational delivery was not necessary. One year followup period revealed no recurrence of arrhythmia.

In conclusion, the non-fluoroscopic catheter ablation guided by electro-anatomical mapping and navigation systems is a safe and applicable treatment method for pregnant women with severe types of arrhythmias.

\section{Conflict of Interests}

Authors declare that they have no conflict of interests.

\section{Ethical Issues}

The current study was approved by the local Ethical Committee of Astana Medical University (Ethics Committee approval No. 1 from 15.03.2018). Informed consent was obtained from the patient.

\section{Financial Support}

The work was carried out without any financial support.

\section{Acknowledgments}

The authors express their gratitude to the hospital management and medical staff, and the patient for their help in carrying out this study.

\section{References}

1. Li JM, Nguyen C, Joglar JA, Hamdan MH, Page RL. Frequency and outcome of arrhythmias complicating admission during pregnancy: experience from a highvolume and ethnically-diverse obstetric service. Clin Cardiol. 2008;31(11):538-541. doi:10.1002/clc.20326

2. Ouzounian JG, Elkayam U. Physiologic changes during normal pregnancy and delivery. Cardiol Clin. 2012;30(3):317-329. doi:10.1016/j.ccl.2012.05.004

3. Hunter S, Robson SC. Adaptation of the maternal heart in pregnancy. Br Heart J. 1992;68(6):540-543. doi:10.1136/ hrt.68.12.540

4. Kumar S, Tedrow UB, Triedman JK. Arrhythmias in adult congenital heart disease: diagnosis and management.
Cardiol Clin. 2015;33(4):571-588, viii. doi:10.1016/j. ccl.2015.07.007

5. Silversides CK, Harris L, Haberer K, Sermer M, Colman JM, Siu SC. Recurrence rates of arrhythmias during pregnancy in women with previous tachyarrhythmia and impact on fetal and neonatal outcomes. Am J Cardiol. 2006;97(8):1206-1212. doi:10.1016/j.amjcard.2005.11.041

6. Joglar JA, Page RL. Antiarrhythmic drugs in pregnancy. Curr Opin Cardiol. 2001;16(1):40-45. doi:10.1097/00001573200101000-00006

7. Priori SG, Blomstrom-Lundqvist C, Mazzanti A, et al. 2015 ESC Guidelines for the management of patients with ventricular arrhythmias and the prevention of sudden cardiac death: The Task Force for the Management of Patients with Ventricular Arrhythmias and the Prevention of Sudden Cardiac Death of the European Society of Cardiology (ESC). Endorsed by: Association for European Paediatric and Congenital Cardiology (AEPC). Eur Heart J. 2015;36(41):2793-2867. doi:10.1093/eurheartj/ehv316

8. Gaita F, Guerra PG, Battaglia A, Anselmino M. The dream of near-zero X-rays ablation comes true. Eur Heart J. 2016;37(36):2749-2755. doi:10.1093/eurheartj/ehw223

9. Bulava A, Hanis J, Eisenberger M. Catheter ablation of atrial fibrillation using zero-fluoroscopy technique: a randomized trial. Pacing Clin Electrophysiol. 2015;38(7):797-806. doi:10.1111/pace.12634

10. Koźluk E, Piątkowska A, Kiliszek M, et al. Catheter ablation of cardiac arrhythmias in pregnancy without fluoroscopy: A case control retrospective study. Adv Clin Exp Med. 2017;26(1):129-134. doi:10.17219/acem/68275

11. Yang PS, Park J, Pak HN. Radiofrequency catheter ablation of incessant atrial tachycardia in pregnant women with minimal radiation exposure: lessons from two case studies. J Cardiol Cases. 2014;9(6):213-216. doi:10.1016/j. jccase.2014.02.001

12. Ferguson JD, Helms A, Mangrum JM, DiMarco JP. Ablation of incessant left atrial tachycardia without fluoroscopy in a pregnant woman. J Cardiovasc Electrophysiol. 2011;22(3):346-349. doi:10.1111/j.1540-8167.2010.01847.x

13. Hsu YC, Chen YP. Right Ventricular outflow Tract (ROVT) Ventricular Tachycardia (VT) in Pregnancy: A Case Report. Intern Med. 2010;21(2):140-143.

14. Wang Y, Chen GZ, Yao Y, et al. Ablation of idiopathic ventricular arrhythmia using zero-fluoroscopy approach with equivalent efficacy and less fatigue: a multicenter comparative study. Medicine (Baltimore). 2017;96(6):e6080. doi:10.1097/md.0000000000006080

15. Hogarth AJ, Graham LN. Normal heart ventricular tachycardia associated with pregnancy: successful treatment with catheter ablation. Indian Pacing Electrophysiol J. 2014;14(2):79-82. doi:10.1016/s0972-6292(16)30733-1 provided the original work is properly cited. 